\title{
-NOTES-
}

\section{A NOTE ON THE MINIMAL REPRESENTATION OF TRIGGERING MATRICES*}

\author{
BY \\ ARTHUR GILL \\ (Department of Electrical Engineering, University of California, Berkeley, Calif.)
}

\begin{abstract}
A triggering matrix is an array of numbers in which each row represents an actuating input of a data-processing system. In this note, upper and lower bounds are given to the minimal form of such a matrix.

In this note the term "triggering set" will refer to any group of differing numbers, each of which serves as an input to a computing or control system. The members of the set, called "trigger-words", have no numerical significance - their only function being the initiation of various processes in the system. It will be assumed that each triggerword initiates a different process, and hence that the only requirement for satisfactory system operation is the ability to differentiate among the words of the set. Typically, a triggering set may be the digital representation of analog quantities to be fed into a recognition system.

In the following discussion, $m$ will denote the number of trigger-words in the set, $n^{\prime}$ the number of digits in each word, and $r$ the "set radix"- the number of values that each digit can assume. $m, n^{\prime}$ and $r$ will be taken as finite. For convenience, a triggering set will be written as a matrix, called the "triggering matrix", in which the element common to the $i$ th row and $j$ th column will represent the $j$ th digit in the $i$ th word. Matrix (1) shown below is an example of a triggering matrix with $m=6, n^{\prime}=9$ and $r=3$.
\end{abstract}

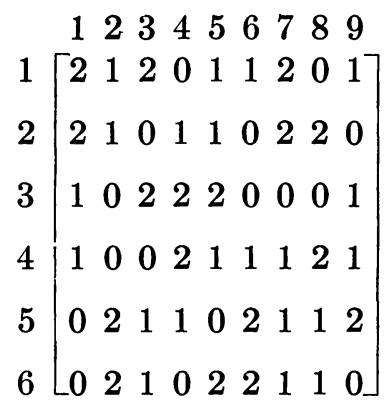

A problem which often arises in data-processing systems with inputs characterizable by triggering matrices, is that of determining the smallest number of digits necessary

*Received November 16, 1959; revised manuscript received January 8, 1960.

The research in this paper was supported by the U. S. Navy under contract with the University of California, Berkeley, California. 
for satisfactory system operation. In terms of the triggering matrix, it is desired to find the smallest number of columns such that the rows are still distinct. Under noisy conditions it is further desired to maintain a specified minimal distance between the rows in order to increase reliability ("distance" having the usual Hamming connotation, extended to codes of radix $r$ ). Deleting the largest number of columns from a triggering matrix such that the distance between any two rows is not less than $d$, results in a matrix which will be called the "minimal triggering matrix consistent with $d$ ". In a previous paper [1] the bounds on this deletion were produced for the special case $r=2$ (i.e., for binary triggering matrices). The following is the extension of the previous results to the general case.

Theorem. Let the dimension of a triggering matrix of radix $r$ and minimal distance $d_{0}$ be $m \times n^{\prime}$. Let the dimension of the corresponding minimal triggering matrix consistent with the minimal distance $d \leq d_{0}$ be $m \times n$. Then

$$
\left[\frac{d-1}{r-1}\right]+\left\{\log _{r} m\right\} \leq n \leq d(m-1),
$$

where $[x]$ denotes the largest integer which is not larger than $x$, and $\{x\}$ the smallest integer which is not smaller than $x$.

The lower bound equals the minimum number of columns which can accommodate radix- $r$ rows with minimal distance $d$. This number can be found by generalizing relationships previously derived in [2] and [3] for the binary minimal distance codes. For that purpose, let $\alpha$ be a sequence of $n$ digits of radix $r$

$$
\alpha=\left(\alpha_{1}, \alpha_{2}, \cdots, \alpha_{n}\right) \quad \alpha_{1}=0, \text { or } 1, \cdots, \text { or } r-1 \text {. }
$$

The sequence $\alpha \ominus \beta$ will be defined by:

$$
\alpha \ominus \beta=\left(\left|\alpha_{1}-\beta_{1}\right|,\left|\alpha_{2}-\beta_{2}\right|, \cdots,\left|\alpha_{n}-\beta_{n}\right|\right) \text {. }
$$

The distance between $\alpha$ and $\beta$, denoted $|\alpha \ominus \beta|$, is then:

$$
|\alpha \ominus \beta|=\sum_{i=1}^{n}\left|\alpha_{i}-\beta_{i}\right| \text {. }
$$

Now, let $M$ be an $m$-word $n$-digit code of radix $r$ and minimal distance $d$. Let $\alpha, \beta(\alpha \neq \beta)$ be any $n$-digit words of radix $r$ which end with $n-[d-1 / r-1]$ zeros. Then:

$$
|\alpha \ominus \beta| \leq(r-1)\left[\frac{d-1}{r-1}\right] \leq d-1
$$

If $\gamma, \delta(\gamma \neq \delta)$ are any elements of $M$, then $\gamma \ominus \alpha$ and $\delta \ominus \beta$ cannot be identical. Consequently:

$$
m \cdot r^{[d-1 / r-1]} \leq r^{n}
$$

or:

$$
n \geq\left[\frac{d-1}{r-1}\right]+\log _{r} m .
$$

Since $n$ must be an integer, the lower bound of the theorem follows.

To verify the upper bound, consider an $m \times n^{\prime}\left\{\log _{2} r\right\}$ binary matrix produced by 
replacing each element in the original triggering matrix with its binary equivalent. This is done in matrix (2) for the ternary triggering matrix (1).

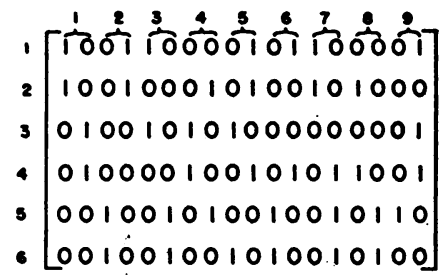

In the new matrix there are $n^{\prime}$ groups of $\left\{\log _{2} r\right\}$ columns, each column group representing a single column in the original matrix. Now, from previous results (see [1]), it is known that the minimal binary matrix consistent with $d$ contains at most $d(m-1)$ columns, and hence at most $d(m-1)$ column groups. Converting these selected column groups back to their original radix- $r$ representation results, then, in a matrix of radix $r$ which contains not more than $d(m-1)$ columns and whose minimal distance is at least $d$. The upper bound is thus verified.

Both the lower and upper bounds in the theorem are achievable with equality. The lower bound is attained, for instance, when $m$ is a power of $r, d=1$, and the triggering set contains all possible radix- $r$ words of length $\log _{r} m$. A triggering matrix in which the upper bound is attained is shown in (3).

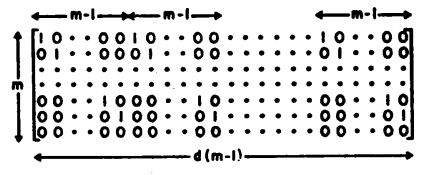

It can be concluded that both bounds on the minimized number of digits are independent of the original number of digits, and that the upper bound is also independent of the number of values which each digit can assume.

\section{BiBLIOGRAPHY}

1. A. Gill, Minimal-scan pattern recognition, IRE Trans. on Information Theory, IT-5, 52-58 (1959)

2. M. Plotkin, Binary codes with specified minimum distance, Research Div. Rept. 51-20, The University of Pennsylvania Moore School of Electrical Engineering, Philadelphia, Pa., 1951

3. D. D. Joshi, A note on upper bounds for minimum distance codes, Information and Control 1, 289-295 (1958) 